\title{
Cold paresis in multifocal motor neuropathy
}

\author{
Dirk C. G. Straver • Jan-Thies H. van Asseldonk • \\ Nicolette C. Notermans • John H. J. Wokke • \\ Leonard H. van den Berg $\cdot$ Hessel Franssen
}

Received: 25 May 2010/Revised: 9 August 2010/Accepted: 11 August 2010/Published online: 28 August 2010

(C) The Author(s) 2010. This article is published with open access at Springerlink.com

\begin{abstract}
Increased weakness during cold (cold paresis) was reported in single cases of multifocal motor neuropathy $(\mathrm{MMN})$. This was unexpected because demyelination is a feature of $\mathrm{MMN}$ and symptoms of demyelination improve, rather than worsen, in cold. It was hypothesized that cold paresis in MMN does not reflect demyelination only, but may indicate the existence of inflammatory nerve lesions with permanently depolarized axons that only just conduct at normal temperature, but fail at lower temperatures. We investigated symptoms of cold paresis in 50 MMN patients, 48 chronic inflammatory demyelinating polyneuropathy (CIDP) patients, 35 progressive spinal muscular atrophy (PSMA) patients, and 25 chronic idiopathic axonal polyneuropathy patients. We also investigated symptoms of increased weakness during warmth (heat paresis). Cold paresis was reported more often than heat paresis. Cold paresis was most frequently reported in MMN. Multivariate analysis indicated that MMN patients had a 4- to 6-fold higher risk of reporting cold paresis than CIDP or PSMA patients. Because cold paresis is not consistent with demyelination, the lesions in MMN may involve other mechanisms than demyelination only. In conclusion, symptoms of cold paresis are common in
\end{abstract}

D. C. G. Straver - J.-T. H. van Asseldonk ·

N. C. Notermans - J. H. J. Wokke - L. H. van den Berg .

H. Franssen

Neuromuscular Disease Group, Department of Neurology,

Rudolf Magnus Institute of Neuroscience,

University Medical Center Utrecht, Heidelberglaan 100,

3584 CX Utrecht, The Netherlands

H. Franssen $(\square)$

Department of Neurology, F02.230,

University Medical Center Utrecht,

PO Box 85500, 3508 GA Utrecht, The Netherlands

e-mail: H.Franssen@umcutrecht.nl peripheral nervous system disorders, particularly in MMN. This supports the above-described hypothesis.

Keywords Multifocal motor neuropathy $\cdot$ Chronic inflammatory demyelinating polyneuropathy $\cdot$ Chronic idiopathic axonal polyneuropathy · Progressive spinal muscular atrophy $\cdot$ Cold paresis

\begin{tabular}{|c|c|}
\hline \multicolumn{2}{|c|}{ Abbreviations } \\
\hline $\mathrm{CB}$ & Conduction block \\
\hline CIAP & Chronic idiopathic axonal polyneuropathy \\
\hline CIDP & $\begin{array}{l}\text { Chronic inflammatory demyelinating } \\
\text { polyneuropathy }\end{array}$ \\
\hline MMN & Multifocal motor neuropathy \\
\hline PSMA & Progressive spinal muscular atrophy \\
\hline
\end{tabular}

\section{Introduction}

The effect of temperature on symptoms of neurological disorders is complicated and has not been fully explored, neither in patients, nor in physiological research. A wellknown effect is the worsening of symptoms in multiple sclerosis after a hot bath [20, 25]. In peripheral neuropathies, similar effects were described. In a patient with chronic inflammatory demyelinating polyneuropathy (CIDP), symptoms considerably increased during fever and in seven patients with various demyelinating neuropathies, electrophysiological signs of conduction block increased after warming and decreased after cooling $[4,8,20]$. This so-called heat paresis may occur in demyelinating disorders and is caused by an unfavorable combination of factors that block saltatory conduction between an active node 
of Ranvier and the node that is next to be activated. First, paranodal demyelination results in current leakage at the node-to-be-activated, so that less current is available to depolarize this node [11]. Secondly, raising temperature will decrease sodium-channel open time at the active node $[6,21]$. This yields a further decrease in the current available to depolarize the node-to-be-activated. If there is just sufficient current available to sustain action potential propagation at a demyelinated internode, an additional decrease in current due to rising temperature may, therefore, induce conduction block as was shown in single demyelinated internodes [19].

In the authors' experience, however, patients with peripheral nervous system disorders often state that weakness during cold increases. This cold paresis is not consistent with the above-described mechanism because that would lead to less weakness in cold. Whether or not cold paresis is really common and if it is related to specific disorders has not been investigated. Furthermore, the mechanisms of cold paresis are not clear. Cold paresis was only reported in single cases of multifocal motor neuropathy (MMN) and in patients with Hirayama disease [11, 23]. To explain cold paresis in MMN, Kaji hypothesized the existence of inflammatory nerve lesions with permanently depolarized axons that only just conduct at normal temperature [11]. At lower temperatures, $\mathrm{Na} / \mathrm{K}$-pump activity decreases due to the thermal reduction of its ATP-ase activity; this may cause additional depolarization, yielding depolarizing conduction block and weakness [9-11]. Recent findings from our group supported the hypothesis of Kaji by showing that cooling indeed induced depolarization of human motor axons, and that this depolarization was best explained by decreased pump-activity [7]. Since, in the future, such axons may possibly be saved from degeneration by neuroprotective treatment, cold paresis may become an important symptom [13, 22, 30].

The present study was not conducted to investigate the mechanism of cold paresis but to assess if symptoms of cold paresis are common in MMN and if they are specific for MMN. If cold paresis were a common symptom, it may instigate further research into its mechanisms. If cold paresis were specific for MMN, it may form a clue for the pathogenesis of MMN, which is currently unknown. We investigated the frequency of symptoms of cold paresis and heat paresis in a large group of patients with MMN. Control groups included CIDP, sporadic progressive spinal muscular atrophy (PSMA), and chronic idiopathic axonal polyneuropathy (CIAP).

\section{Materials and methods}

\section{Patients}

A cross-sectional study was performed by sending a questionnaire to 60 patients with MMN, 60 with CIDP, 50 with sporadic PSMA, and 35 patients with CIAP. All patients had weakness in arm or leg muscles on neurological examination. The questionnaire was returned by $83 \%$ of MMN, $80 \%$ of CIDP, $70 \%$ of PSMA, and $71 \%$ of CIAP patients. For each group, the patients who did not return the questionnaire did not differ significantly from the patients who returned the questionnaire with respect to sex, age, and disease duration (data not shown). Patient characteristics are presented in Table 1. Two patients in the PSMA group had Hirayama disease. The patients attended the outpatient clinics of the University Medical Center in Utrecht, a tertiary referral center for neuromuscular disorders in the Netherlands. Diagnoses were based on criteria sets for MMN [28], CIDP [1], sporadic PSMA [29], and CIAP [16]. In these sets, the criteria for conduction block were adapted [27, 28]. All MMN patients were on intravenous immunoglobulins (IVIg) maintenance treatment. Twenty-eight patients with CIDP were treated with IVIg, with 20 patients responding favorably. Twelve patients with CIDP were treated with steroids, with 11 patients responding favorably. Of the remaining CIDP patients, two were successfully treated with cyclophosphamide and six were not treated. None of the patients had concomitant diseases of the central or peripheral nervous system. All participants gave informed consent to participate in the study, which was approved by the Medical Ethical Committee of the University Medical Center Utrecht.

Table 1 Baseline characteristics

\begin{tabular}{llllcrrr}
\hline Patient group & $\begin{array}{l}\text { Male } \\
(\%)\end{array}$ & $\begin{array}{l}\text { Age } \\
\text { (years) }\end{array}$ & $\begin{array}{l}\text { Disease duration } \\
\text { (years) }\end{array}$ & $\begin{array}{l}\text { Weakness } \\
\text { arm }(\%)\end{array}$ & $\begin{array}{l}\text { Weakness } \\
\text { leg }(\%)\end{array}$ & $\begin{array}{l}\text { CB arm } \\
\text { nerves }(\%)\end{array}$ & $\begin{array}{l}\text { CB leg } \\
\text { nerves }(\%)\end{array}$ \\
\hline MMN $(n=50)$ & 82 & $51(33-74)$ & $10(2-39)$ & 100 & 68 & 68 & 24 \\
CIDP $(n=48)$ & 58 & $54(22-82)$ & $4(0-32)$ & 88 & 96 & 44 & 19 \\
PSMA $(n=35)$ & 86 & $61(35-83)$ & $12(4-30)$ & 86 & 51 & 0 & 0 \\
CIAP $(n=25)$ & 80 & $75(61-89)$ & $13(4-21)$ & 28 & 100 & 0 \\
\hline
\end{tabular}

Age and disease duration are given as median (range)

$C B$ conduction block; $M M N$ multifocal motor neuropathy; $C I D P$ chronic inflammatory demyelinating polyneuropathy; $P S M A$ progressive spinal muscular atrophy; CIAP chronic idiopathic axonal polyneuropathy 
Table 2 Percentage of patients exposed to cooling

\begin{tabular}{llllll}
\hline Patient group & Any & Bathing & Weather & Swimming & Cold pack \\
\hline MMN & 96 & 20 & 96 & 30 & 20 \\
CIDP & 90 & 23 & 88 & 29 & 8 \\
PSMA & 97 & 17 & 97 & 26 & 9 \\
CIAP & 72 & 8 & 72 & 8 & 4 \\
\hline
\end{tabular}

$M M N$ multifocal motor neuropathy; CIDP chronic inflammatory demyelinating polyneuropathy; PSMA progressive spinal muscular atrophy; CIAP chronic idiopathic axonal polyneuropathy

The results of the last nerve conduction studies, performed prior to the questionnaire, were reviewed for presence of definite conduction block (segmental reduction in negative compound muscle action potential area of at least $50 \%$ in any nerve) and probable conduction block (segmental reduction in negative compound muscle action potential amplitude of at least $30 \%$ in an arm nerve) [27]. Conduction studies included the median nerve (recording from the thenar and flexor carpi radialis muscle), ulnar nerve, radial nerve, and musculocutaneous nerve up to Erb's point, and the peroneal and tibial nerves up to the knee [27]. These nerves were studied bilaterally, except for five patients with CIDP and the patients with CIAP who were studied unilaterally. Conduction block in arm or leg nerves was found in all MMN patients, in $82 \%$ of CIDP patients, and in none of the PSMA or CIAP patients (Table 1). MMN patients had conduction block in arm nerves more often than CIDP patients.

\section{Questionnaire}

The questionnaire consisted of temperature-related and weakness-related items. Questions concerned the year preceding the questionnaire to reduce recall bias. First, we asked if symptoms of weakness and conditions of cooling or warming had occurred. Conditions of cooling included a stay in cold weather, the use of cold packs or taking a cold shower, bath or swim. Conditions of warming included a stay in hot weather, the use of hot packs or electric blankets, rinsing the hands in hot water while washing dishes or taking a warm or hot shower, bath or swim. If symptoms of weakness and conditions of cooling or warming had occurred, we asked if weakness increased during cooling (cold paresis) or warming (heat paresis). Exposure to cooling or warming and increase in weakness were entered as dichotomous variables. Prior to the study, the questionnaire was tested for inconsistencies and feasibility by a randomly chosen sample of 20 patients who visited our outpatient clinic for neuromuscular disorders with symptoms of weakness and who were not included in this study.

\section{Statistical analysis}

We calculated the frequency of symptoms of cold (or heat) paresis in arms with established weakness and in legs with established weakness. We determined if: (1) exposure to cooling and warming differed between patient groups, and (2) the percentage of patients with CIDP, PSMA, or CIAP reporting symptoms of cold or heat paresis differed from the percentage of patients with MMN reporting symptoms of cold or heat paresis (Chi-square tests).

Next, univariate and multivariate binary logistic regression analyses were performed. The determinant of interest was presence of MMN with CIDP, PSMA, and CIAP as reference groups. Outcome variables included: (1) symptoms of cold paresis in arms or legs, (2) symptoms of cold paresis in arms, (3) symptoms of cold paresis in legs, (4) symptoms of heat paresis in arms or legs, (5) symptoms of heat paresis in arms, and (6) symptoms of heat paresis in legs. Age and disease duration were assessed as continuous variables. The possible confounders sex, age (in years), and disease duration (in years) at the time of the questionnaire were added in the multivariate models.

Version 15.0.1 of the SPSS statistical software program (Chicago, IL, USA) was used for all analyses. $p$ values $<0.05$ were considered to be significant.

\section{Results}

Exposure to cooling or warming did not differ significantly between MMN, CIDP, and PSMA, but CIAP patients were significantly less often exposed to cooling than MMN and

Table 3 Percentage of patients exposed to warming

\begin{tabular}{lrllllll}
\hline Patient group & Any & Bathing & Weather & Sauna & Hot pack & Electric blanket & Dish-washing \\
\hline MMN & 100 & 90 & 82 & 26 & 12 & 22 & 62 \\
CIDP & 98 & 92 & 90 & 19 & 2 & 3 & 65 \\
PSMA & 97 & 97 & 80 & 20 & 3 & 14 & 54 \\
CIAP & 96 & 76 & 60 & 0 & 0 & 20 & 52 \\
\hline
\end{tabular}

MMN multifocal motor neuropathy; CIDP chronic inflammatory demyelinating polyneuropathy; PSMA progressive spinal muscular atrophy; CIAP chronic idiopathic axonal polyneuropathy 
Table 4 Percentage of patients reporting cold and heat paresis

\begin{tabular}{llccc}
\hline Patient group & Cold paresis & $p$ value & Heat paresis & $p$ value \\
\hline MMN & 83 & - & 18 & - \\
CIDP & 44 & $<0.001$ & 26 & $\mathrm{~ns}$ \\
PSMA & 50 & 0.001 & 6 & $\mathrm{~ns}$ \\
CIAP & 44 & 0.002 & 17 & $\mathrm{~ns}$ \\
\hline
\end{tabular}

$p$ values refer to comparison with $\mathrm{MMN}$

$M M N$ multifocal motor neuropathy; CIDP chronic inflammatory demyelinating polyneuropathy; PSMA progressive spinal muscular atrophy; CIAP chronic idiopathic axonal polyneuropathy; $n s$ not significant

PSMA patients (Tables 2, 3). Exposure to warming was more common than exposure to cooling and cold weather was the most common exposure to cooling. Despite this, patients reported cold paresis more often than heat paresis (Table 4).

Cold paresis in arms or legs was reported more frequently in MMN than in CIDP, PSMA, and CIAP (Table 4). Multivariate analysis showed that these differences were independent of sex, age, and disease duration, except for CIAP (Table 5). Patients with MMN had a fivefold higher risk of symptoms of cold paresis than patients with CIDP, and a sixfold higher risk than patients with PSMA. Multivariate analysis for symptoms of cold paresis in arms and legs separately, showed that these differences were still significant for the arms but not for the legs (Table 5).

Heat paresis in arms or legs was not significantly more or less frequently reported in MMN than in CIDP, PSMA and CIAP (Table 4). Neither univariate nor multivariate analysis showed any significant differences between MMN and the other patient groups (data not shown).

\section{Discussion}

The present study demonstrated that cold paresis was experienced by a substantial proportion of patients with
MMN, CIDP, PSMA, and CIAP. Cold paresis was more frequently reported than heat paresis. Cold paresis was more frequently reported in MMN than in CIDP, PSMA, or CIAP. Multivariate analysis indicated that MMN patients had a 4- to 6-fold greater risk of experiencing cold paresis than CIDP or PSMA patients. Because MMN predominantly affects arm nerves [27], we analyzed symptoms of cold paresis in arms and legs separately; this showed that these differences were still significant for the arms but not for the legs.

In MMN, electrophysiological, pathological, and immunological studies suggest that several mechanisms may be involved in the unique process that results in asymmetric loss of strength and muscular atrophy. Motor nerve conduction studies may show conduction block and slowing consistent with demyelination in the same segment; however, conduction velocity may also be normal in segments with motor conduction block [27, 28]. Some pathological studies of nerve branches containing motor and sensory axons showed demyelination but others only showed loss of axons [2, 5, 12, 24]. Needle electromyography studies showed extensive neurogenic abnormalities in non-atrophic as well as in atrophic muscles [26]. Excitability studies showed focal depolarization or hyperpolarization of motor axons of which the significance is not clear $[14,17]$. In many MMN patients, antibodies against epitopes presented on the axolemma are found, and a recent study showed that these anti-GM1 antibodies are associated with loss of motor axons [3]. Thus, whereas demyelination clearly occurs in MMN, mechanisms leading to primary axonal degeneration may possibly occur as well. It is not clear if the conduction block is due to unfavorable effects of paranodal demyelination, blocking of impulse generation at the node, or both. Because MMN may lead to demyelination as well as loss of motor axons, we have chosen CIDP, PSMA, and CIAP as control groups in order to make a comparison to peripheral nervous system disorders that are also characterized by weakness: CIDP is characterized mainly by demyelination of motor

Table 5 Risk of cold paresis in MMN versus other disorders

\begin{tabular}{|c|c|c|c|c|c|c|}
\hline Patient groups & Arm and leg & Odds ratio & $p$ value & Arm & Odds ratio & $p$ value \\
\hline \multirow[t]{2}{*}{ MMN-CIDP } & Univariate & $6.3(2.4-16.6)$ & $<0.001$ & Univariate & $6.9(2.4-20.0)$ & $<0.001$ \\
\hline & Multivariate & $4.7(1.7-13.4)$ & 0.003 & Multivariate & $5.3(1.7-16.7)$ & 0.005 \\
\hline \multirow[t]{2}{*}{ MMN-PSMA } & Univariate & $5.0(1.8-13.8)$ & 0.002 & Univariate & $3.4(1.1-10.3)$ & 0.03 \\
\hline & Multivariate & $5.7(1.8-18.1)$ & 0.003 & Multivariate & $3.8(1.04-14.3)$ & 0.04 \\
\hline \multirow[t]{2}{*}{ MMN-CIAP } & Univariate & $6.3(1.9-20.8)$ & 0.003 & & & \\
\hline & Multivariate & $4.0(0.4-36.5)$ & ns & & & \\
\hline
\end{tabular}

Odds ratios are given with $95 \%$ confidence intervals. Multivariate analysis was adjusted for age, sex, and disease duration. Cold paresis in arm muscles in the CIAP group was not analyzed because only two patients had upper limb weakness and were exposed to cold

$M M N$ multifocal motor neuropathy; CIDP chronic inflammatory demyelinating polyneuropathy; PSMA progressive spinal muscular atrophy; CIAP chronic idiopathic axonal polyneuropathy; $n s$ not significant 
and sensory axons, PSMA by loss of motor axons, and CIAP by length-dependent loss of motor and sensory axons.

Cold paresis was reported by two-thirds of patients with distal upper limb muscular atrophy (Hirayama disease), a disorder affecting peripheral motor neurons in the anterior horn of the cervical cord, which results in denervation of hand muscles [23]. Nerve conduction studies in 11 patients with Hirayama disease, and one patient with hypothenar atrophy due to ulnar neuropathy, showed that cold induced excessive conduction delay and waning of the compound muscle action potential during $20-\mathrm{Hz}$ repetitive stimulation $[15,18]$. These findings were attributed to an increased sensitivity of reinnervated muscle fibers to develop depolarizing conduction block in cold.

Cold paresis in MMN was suggested to be related to the existence of inflammatory nerve lesions where axons are depolarized but just able to conduct impulses at ambient temperature [11]. At these lesions, thermal reduction of $\mathrm{Na} /$ $\mathrm{K}$-pump activity due to cooling may induce further depolarization to a point where impulse conduction fails. Some findings may support this hypothesis. First, animal models of inflammatory spinal root lesions indicated that inflammation may induce nitric oxide-mediated mitochondrial dysfunction, energy depletion of the ATP-ase dependent $\mathrm{Na} / \mathrm{K}$-pump, and axonal depolarization [13]. Secondly, excitability studies in MMN indirectly suggested that axons at the site of block may possibly be depolarized [14, 17]. Thirdly, cooling of normal human motor axons results in their depolarization, probably due to decreased $\mathrm{Na}$ /K-pump activity [7]. Some of the steps in this hypothesis were corroborated by experiments, but it must be emphasized, however, that its entire sequence has not been proven. Moreover, some experiments were carried out in central nervous system neurons so that the results possibly cannot be extrapolated to peripheral motor neurons.

Alternatively, cold paresis in MMN may be caused by development of conduction block in reinnervated muscle fibers due to the lower temperature $[15,18]$. The possibility that this mechanism, which may be similar to that described in Hirayama disease, occurs in MMN is supported by several studies. Extensive needle electromyography studies in MMN patients showed signs of reinnervation, indicating collateral sprouting, in non-atrophic as well as in atrophic muscles [26]. Some pathological studies of motor nerves in MMN have shown prominent loss of axons and little demyelination; the loss of axons may lead to collateral sprouting and reinnervated muscle fibers [24].

In conclusion, symptoms of cold paresis occur in several peripheral nervous system disorders, but are considerably more common in MMN. Because the present study only assessed subjective symptoms of weakness, it is worthwhile to investigate if cold also induces an objective increase of weakness in MMN.

Acknowledgments J. T. H. van Asseldonk and D. C. G. Straver were supported by grants from the Prinses Beatrix Fonds.

Conflict of interest The authors declare that they have no conflict of interest.

Open Access This article is distributed under the terms of the Creative Commons Attribution Noncommercial License which permits any noncommercial use, distribution, and reproduction in any medium, provided the original author(s) and source are credited.

\section{References}

1. AAN (1991) Research criteria for diagnosis of chronic inflammatory demyelinating polyneuropathy (CIDP). Neurology 41:617-618

2. Auer RN, Bell RB, Lee MA (1989) Neuropathy with onion bulb formations and pure motor manifestations. Can J Neurol Sci 16:194-197

3. Cats EA, van der Pol WL, Piepers S, Franssen H, Jacobs BC, Van den Berg-Vos RM, Kuks JJ, van Doorn P, van Engelen BG, Verschuuren JJ, Wokke JH, Veldink JH, van den Berg LH (2010) Correlates of outcome and response to IVIg in 88 patients with multifocal motor neuropathy. Neurology (in press)

4. Chaudhry V, Crawford TO, DeRossett SE (1993) Thermal sensitivity in demyelinating neuropathy. Muscle Nerve 16:301-306

5. Corbo M, Abouzahr MK, Latov N, Iannaccone S, Quattrini A, Nemni R, Canal N, Hays AP (1997) Motor nerve biopsy studies in motor neuropathy and motor neuron disease. Muscle Nerve 20:15-21

6. Frankenhäuser B, Moore LE (1963) The effect of temperature on the sodium and potassium permeability changes in myelinated nerve fibres of Xenopus laevis. J Physiol 169:431-437

7. Franssen H, Gebbink TA, Wokke JH, van den Berg LH, Van Schelven LJ (2010) Is cold paresis related to axonal depolarization? J Peripher Nerv Syst (in press)

8. Franssen H, Wieneke GH, Wokke JH (1999) The influence of temperature on conduction block. Muscle Nerve 22:166-173

9. Gordon TR, Kocsis JD, Waxman SG (1990) Electrogenic pump $\left(\mathrm{Na}^{+} / \mathrm{K}^{+}\right.$-ATP-ase) activity in rat optic nerve. Neuroscience 37:829-837

10. Hodgkin AL, Keynes RD (1955) Active transport of cations in giant axons from Sepia and Loligo. J Physiol 128:28-60

11. Kaji R (2003) Physiology of conduction block in multifocal motor neuropathy and other demyelinating neuropathies. Muscle Nerve 27:285-296

12. Kaji R, Oka N, Tsuji T, Mezaki T, Nishio T, Akiguchi I, Kimura J (1993) Pathological findings at the site of conduction block in multifocal motor neuropathy. Ann Neurol 33:152-158

13. Kapoor R, Davies M, Blaker PA, Hall SM, Smith KJ (2003) Blockers of sodium and calcium entry protect axons from nitric oxide-mediated degeneration. Ann Neurol 53:174-180

14. Kiernan MC, Guglielmi JM, Kaji R, Murray NM, Bostock H (2002) Evidence for axonal membrane hyperpolarization in multifocal motor neuropathy with conduction block. Brain 125:664-675

15. Kijima M, Hirayama K, Nakajima Y (2002) Symptomatological and electrophysiological study on cold paresis in juvenile 
muscular atrophy of distal upper extremity (Hirayama's disease). Rinsho Shinkeigaku 42:841-848

16. Notermans NC, Wokke JH, Franssen H, Van der GY, Vermeulen M, Van den Berg LH, Bar PR, Jennekens FG (1993) Chronic idiopathic polyneuropathy presenting in middle or old age: a clinical and electrophysiological study of 75 patients. J Neurol Neurosurg Psychiatry 56:1066-1071

17. Priori A, Bossi B, Ardolino G, Bertolasi L, Carpo M, NobileOrazio E, Barbieri S (2005) Pathophysiological heterogeneity of conduction blocks in multifocal motor neuropathy. Brain 128:1642-1648

18. Rack PM, Fox JE (1987) The effects of cold on a partially denervated muscle. J Neurol Neurosurg Psychiatry 50:460-464

19. Rasminsky M (1973) The effects of temperature on conduction in demyelinated single nerve fibers. Arch Neurol 28:287-292

20. Rutkove SB (2001) Effects of temperature on neuromuscular electrophysiology. Muscle Nerve 24:867-882

21. Schwarz JR, Eikhof G (1987) Na currents and action potentials in rat myelinated nerve fibres at 20 and $37^{\circ} \mathrm{C}$. Pflugers Arch 409:569-577

22. Stys PK (2005) General mechanisms of axonal damage and its prevention. J Neurol Sci 233:3-13

23. Tashiro K, Kikuchi S, Itoyama Y, Tokumaru Y, Sobue G, Mukai E, Akiguchi I, Nakashima K, Kira J, Hirayama K (2006) Nationwide survey of juvenile muscular atrophy of distal upper extremity (Hirayama disease) in Japan. Amyotroph Lateral Scler $7: 38-45$
24. Taylor BV, Dyck PJ, Engelstad J, Gruener G, Grant I, Dyck PJ (2004) Multifocal motor neuropathy: pathologic alterations at the site of conduction block. J Neuropathol Exp Neurol 63:129-137

25. Uhthoff W (1890) Untersuchungen über die bei der Multiplen Herdsklerose vorkommenden Augenstörungen. Arch Psychiatr Nerverkr 21:303-410

26. Van Asseldonk JTH, van den Berg LH, Kalmijn S, Van den BergVos RM, Polman CH, Wokke JHJ, Franssen H (2006) Axon loss is an important determinant of weakness in multifocal motor neuropathy. J Neurol Neurosurg Psychiatry 77:743-750

27. Van Asseldonk JTH, van den Berg LH, Van den Berg-Vos RM, Wieneke GH, Wokke JH, Franssen H (2003) Demyelination and axonal loss in multifocal motor neuropathy: distribution and relation to weakness. Brain 126:186-198

28. Van den Berg-Vos RM, Franssen H, Wokke JH, van Es HW, van den Berg LH (2000) Multifocal motor neuropathy: diagnostic criteria that predict the response to immunoglobulin treatment. Ann Neurol 48:919-926

29. Van den Berg-Vos RM, Visser J, Franssen H, de Visser M, de Jong JM, Kalmijn S, Wokke JH, van den Berg LH (2003) Sporadic lower motor neuron disease with adult onset: classification of subtypes. Brain 126:1036-1047

30. Waxman SG (1995) Sodium channel blockade by antibodies: a new mechanism of neurological disease? Ann Neurol 37:421-423 